\title{
Limited genetic diversity in Salmonella enterica Serovar Enteritidis
} PTI3

\author{
Adam B Olson ${ }^{1}$, Ashleigh K Andrysiak ${ }^{1,4}$, Dobryan M Tracz ${ }^{1}$, Jean Guard- \\ Bouldin $^{2}$, Walter Demczuk ${ }^{1}$, Lai-King Ng1,4, Anne Maki ${ }^{3}$, Frances Jamieson ${ }^{3}$ \\ and Matthew W Gilmour*1,4
}

Address: ${ }^{1}$ National Microbiology Laboratory, Public Health Agency of Canada, Winnipeg, MB, Canada, ${ }^{2}$ United States Department of Agricultural, Agricultural Research Service, Athens, GA, USA, ${ }^{3}$ Ontario Central Public Health Laboratory, Ministry of Health and Long-Term Care, Toronto, ON, Canada and ${ }^{4}$ Department of Medical Microbiology, University of Manitoba, Winnipeg, MB, Canada

Email: Adam B Olson - Adam_Olson@phac-aspc.gc.ca; Ashleigh K Andrysiak - Ashleigh_Andrysiak@phac-aspc.gc.ca; Dobryan M Tracz - Dobryan_Tracz@phac-aspc.gc.ca; Jean Guard-Bouldin - Jean.Guard.Bouldin@ars.usda.gov;

Walter Demczuk - Walter_Demczuk@phac-aspc.gc.ca; Lai-King Ng - Lai_King_Ng@phac-aspc.gc.ca; Anne Maki - Anne.Maki@moh.gov.on.ca; Frances Jamieson - Frances.Jamieson@moh.gov.on.ca; Matthew W Gilmour* - matt.gilmour@gmail.com

* Corresponding author

Published: I October 2007

BMC Microbiology 2007, 7:87 doi:10.1/86/|47|-2/80-7-87
Received: 12 June 2007

Accepted: I October 2007

This article is available from: http://www.biomedcentral.com/147I-2180/7/87

(C) 2007 Olson et al; licensee BioMed Central Ltd.

This is an Open Access article distributed under the terms of the Creative Commons Attribution License (http://creativecommons.org/licenses/by/2.0), which permits unrestricted use, distribution, and reproduction in any medium, provided the original work is properly cited.

\begin{abstract}
Background: Salmonella enterica serovar Enteritidis has emerged as a significant foodborne pathogen throughout the world and is commonly characterized by phage typing. In Canada phage types (PT) 4, 8 and 13 predominate and in 2005 a large foodborne PTI 3 outbreak occurred in the province of Ontario. The ability to link strains during this outbreak was difficult due to the apparent clonality of PTI 3 isolates in Canada, as there was a single dominant pulsed-field gel electrophoresis (PFGE) profile amongst epidemiologically linked human and food isolates as well as concurrent sporadic strains. The aim of this study was to perform comparative genomic hybridization (CGH), DNA sequence-based typing (SBT) genomic analyses, plasmid analyses, and automated repetitive sequence-based PCR (rep-PCR) to identify epidemiologically significant traits capable of subtyping S. Enteritidis PTI3.

Results: CGH using an oligonucleotide array based upon chromosomal coding sequences of $S$. enterica serovar Typhimurium strain LT2 and the Salmonella genomic island I successfully determined major genetic differences between S. Typhimurium and S. Enteritidis PTI3, but no significant strain-to-strain differences were observed between S. Enteritidis PTI 3 isolates. Individual loci (safA and fliC) that were identified as potentially divergent in the $\mathrm{CGH}$ data set were sequenced in a panel of $S$. Enteritidis strains, and no differences were detected between the PTI3 strains. Additional sequence-based typing was performed at the fimA, $m d h$, manB, cyaA, citT, caiC, dmsA, ratA and STM0660 loci. Similarly, no diversity was observed amongst PTI 3 strains. Variation in plasmid content between PTI3 strains was observed, but macrorestriction with Bglll did not identify further differences. Automated rep-PCR patterns were variable between serovars, but S. Enteritidis PTI 3 strains could not be differentiated.

Conclusion: None of the methods identified any significant variation between PTI 3 strains. Greater than I I,300 base pairs of sequence for each of seven S. Enteritidis PT I 3 strains were analyzed without detecting a single polymorphic site, although diversity between different phage types of $S$. Enteritidis was observed. These data suggest that Canadian S. Enteritidis PTI 3 strains are highly related genetically.
\end{abstract}




\section{Background}

Salmonella enterica serovar Enteritidis (S. Enteritidis) is a foodborne pathogen transmitted to humans predominately through contaminated eggs and other poultry food products [1]. S. Enteritidis was an infrequently reported serovar until the mid- to late 1980's when it emerged as a common cause of salmonellosis in European countries and then worldwide [2-4]. By the 1990's S. Enteritidis replaced $S$. enterica serovar Typhimurium (S. Typhimurium) as the most common serovar isolated from humans in many countries $[5,3,6,4,7]$. In Canada, $S$. Enteritidis has been among the top three reported Salmonella serovars resulting in human disease since 1998, accounting for between $12-21 \%$ of infections caused by Salmonella [8]. S. Enteritidis is subtyped by a phage typing scheme implemented by Ward et al., [9], and this method has identified regionally endemic $S$. Enteritidis subtypes as well fluctuations in the predominate subtypes. For example, $S$. Enteritidis phage type 13 (PT13) was the eighth most common phage type identified in Canada from 1982-1992, but by 1998 PT13 was ranked the third most common and has subsequently remained one of the top five most prevalent phage types [8].

Phage typing of $S$. Enteritidis utilizes a set of sixteen bacteriophage to generate a lytic pattern to group strains [9]. Typing methods based upon comparisons of whole genomic DNA, plasmid DNA or specific genetic determinants have also been used in place of phage typing or as supplementary techniques. The methods most frequently used to subtype $S$. Enteritidis include pulsed-field gel electrophoresis (PFGE), plasmid profiling and restrictionhybridization based ribotyping, which have each been applied with varying degrees of success [10-16]. A single PFGE macrorestriction profile often predominated in strains of the same phage type or even amongst multiple phage types $[10,13,17-19]$. Alternatively, a group of strains of the same phage type can have multiple restriction patterns [20,21]. Ribotyping based upon restriction analysis of the rRNA operon has utilized several schemes, such as PvuII or a double digestion with PstI and SphI [22]. The latter combination has been used to subtype strains of the same phage type, however, this discrimination was not always epidemiologically significant [17]. Plasmid profiles have indicated divergence between strains represented by the presence or absence of various low molecular weight plasmids and the 55 to $60 \mathrm{kbp} S$. Enteritidis virulence plasmid $[11,12,23]$, which is similar to the 94 kbp pSLT virulence plasmid of S. Typhimurium [24]. Combinations of these methods have been successfully used to subtype outbreak from non-outbreak strains of $S$. Enteritidis $[25,11,13,15,18]$.

DNA microarray-based comparative genomic hybridization (CGH) and sequence-based typing (SBT) have recently been used to determine genetic relatedness between $S$. Enteritidis strains, and there was generally a lack of diversity for strains of the same phage type [26,24,27-29]. SBT of 24 clinical and poultry-related $S$. Enteritidis strains in a scheme comprised of segments of the 16 S rRNA, manB, glnA and $p d u F$ loci detected 2, 37, 3 and 3 polymorphic sites, respectively [28]. Similarly, a SBT scheme evaluating $\operatorname{manB}, m d h$ and fim $A$ encoded by 7 $S$. Enteritidis strains revealed 2, 2 and 0 polymorphic sites, respectively [26]. Typing of the misL, spaM and spaN loci encoding cell surface-associated proteins did not identify differences in four PT13 strains, but identified 2 polymorphic sites in PT4 [29]. CGH analyses of a diverse collection of $27 \mathrm{~S}$. Enteritidis strains using a microarray composed of $S$. Typhimurium, $S$. Typhi, S. Paratyphi A and $S$. Enteritidis PT4 probes identified few genetic differences between these strains, including strains isolated before the emergence of $S$. Enteritidis as a major foodborne Salmonella [24]. The genetic differences were predominantly at phage-encoding loci (ST64B and Fels-2) but no significant delineation amongst $S$. Enteritidis strains of the same phage type was observed [24]. CGH analyses of PT13a and PT4 strains similarly identified no significant strain-to-strain variation, and these phage types were also distinguished by ST64B and Fels bacteriophage genetic content [27].

S. Enteritidis PT13 was identified in a large foodborne outbreak in the Canadian province of Ontario in 2005. Over 700 cases of gastroenteritis were reported between October and December 2005 and these were associated with the consumption of contaminated mung bean sprouts. A single PFGE profile (SENXAI.0038; SENBNI.0016) predominated amongst the outbreakassociated strains, and this profile was also seen in concurrent and preceding sporadic human-clinical and poultryrelated isolates. With the apparent clonality of PT13 isolates, it was difficult to support epidemiological links during the outbreak using PFGE-based typing. The aim of this study was to perform CGH and SBT genomic analyses in parallel with other typing methodologies to identify epidemiologically significant markers for subtyping $S$. Enteritidis PT13.

\section{Results}

In response to the large outbreak of $S$. Enteritidis PT13, all $S$. Enteritidis PT13 strains with PFGE data reported to the National Microbiology Laboratory were retrospectively analyzed. This set of 32 strains comprised sporadic human-clinical isolates and also poultry-related agri-food isolates submitted by provincial public health laboratories in Alberta, British Columbia, Ontario and Québec. Two PFGE patterns were observed: 30 strains had the XbaI macrorestriction profile SENXAI.0038 and 2 strains produced the related pattern SENXAI.0062 (Fig. 1). PFGE 
SENXAI.0038

SENXAI.0062

\section{Figure I}

Pulsed-field gel electrophoresis of S. Enteritidis PTI 3 using $X$ bal. Two macrorestriction patterns (SENXAI) were observed, and are presented with high to low molecular weight fragments from left to right.

with $B \ln I$ was performed for 19 of these strains and all were pattern SENBNI.0016. The inability to differentiate strains by PFGE necessitated the need to attempt additional subtyping methodologies for the differentiation of both outbreak and sporadic S. Enteritidis PT13 strains.

\section{Comparative genomic hybridization}

Microarray-based CGH was performed on S. Enteritidis strains to identify genetic differences between strains that would potentially serve as molecular markers for subtyping S. Enteritidis PT13 strains. An oligonucleotide array representing all coding sequences from the sequenced genome of $S$. Typhimurium LT2 and all coding sequences from SGI1 [30] was used in two-colour CGH experiments with $S$. Typhimurium LT2 genomic DNA as a reference and $S$. Enteritidis genomic DNA as the test strain. Seven PT13 strains were analyzed, including: human isolate 056733 associated with the foodborne outbreak; strain 056746 isolated from mung bean sprouts presumptively identified as the causative food agent; and five other sporadic PT13 strains isolated from human and poultry sources that were recovered concurrently with the outbreak or previously in other regions of Canada (see Table

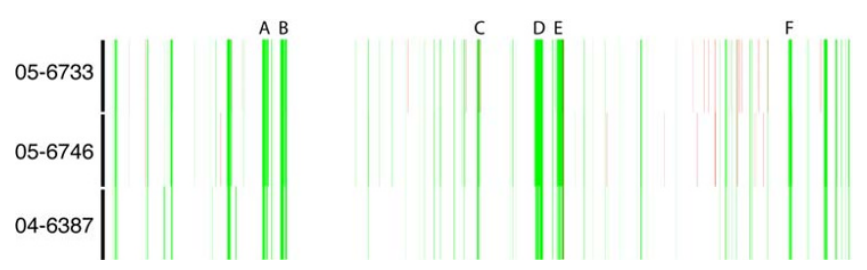

\section{Figure 2}

DNA microarray-based comparative genomics of S. Enteritidis PTI3. Array probes represent the linear order of $S$. Typhimurium LT2 coding sequences from left to right, with the custom Salmonella genomic island I (SGII) at the far-left side. White denotes similarity to LT2, green denotes putative divergence and red represents putative duplication or copy number change. Clusters of bacteriophage-related determinants that are divergent in $S$. Enteritidis compared to $S$. Typhimurium: A, STM893-929 (Fels-I prophage); B, STMI005-I024 (Gifsy-2 prophage); C, STM2230-2240 (putative phage); D, STM2589-2636 (Gifsy-I prophage); E, STM2732-2772 (Fels-2 prophage); F, STM4 I 98-42I 8 (putative phage).
1). The CGH analyses of PT13 strains individually indicated the genetic content of the strains in comparison to $S$. Typhimurium LT2, and the majority of the differences corresponded to genes encoded by bacteriophages Fels-1, Fels-2, Gifsy-1 and Gifsy-2, and also putative phagerelated coding sequences STM2230-2240 and STM41984217 (Fig. 2). The oligonucleotides probes for SGI1 indicated that this determinant was absent in all strains, and accordingly, all examined $S$. Enteritidis strains were sensitive to antibiotics. The relative genetic content of $S$. Enteritidis strains identified by microarray analysis could also be used to compare between PT13 strains, and few coding sequences were putatively divergent between PT13 strains. DNA sequencing was performed at fliC and safA (both of which were putatively divergent in the CGH dataset), and each locus was identical for all strains PT13 strains (Table 1). These data suggested that differences in genetic content between PT13 strains in the CGH dataset were partly due to technical variation rather than true biological variation.

\section{Sequence-based typing}

Due to the lack of observable differences in genetic content between PT13 using CGH, additional molecular methods were attempted to differentiate these strains. A SBT scheme targeting $m a n B$, mah and fimA [26] did not identify sequence type differences amongst $S$. Enteritidis PT13 (Table 1). A single nucleotide polymorphism (SNP) was observed at the manB locus for both $S$. Enteritidis PT1 and PT4 compared to $S$. Enteritidis PT13. Previously, multiple SNPs have been observed between $S$. Enteritidis strains at the cyaA locus [31] and additional comparative analyses of $S$. Enteritidis phenotypic subpopulations that were descended from a common parent by whole genome mutational mapping identified polymorphisms, including small deletions, non-synonymous amino acid changes, and altered terminal codons [32]. From this data set, we sequenced for each strain in our $S$. Enteritidis panel the ratA, citT, dmsA, caiC and STM0660 loci encoding membrane proteins or metabolic cellular functions, and similarly no polymorphisms were observed between PT13 strains. A total of $11,390 \mathrm{bp}$, including the fliC and safA loci examined after CGH analyses, was sequenced for each strain without observing any genetic differences between the $S$. Enteritidis PT13 strains. A total of 7 SNPs were found between $S$. Enteritidis PT1 and PT4 compared to $S$. Enteritidis PT13, and a multitude of polymorphic sites between S. Typhimurium LT2 and S. Enteritidis PT13 were observed (Table 1).

\section{Plasmid content and plasmid RFLP}

The population and characteristics of plasmids harbored by Salmonella has been used as a means to subtype these organisms [11]. Plasmid content was determined for the $S$. Enteritidis strains, as well as $S$. Typhimurium strain LT2, 


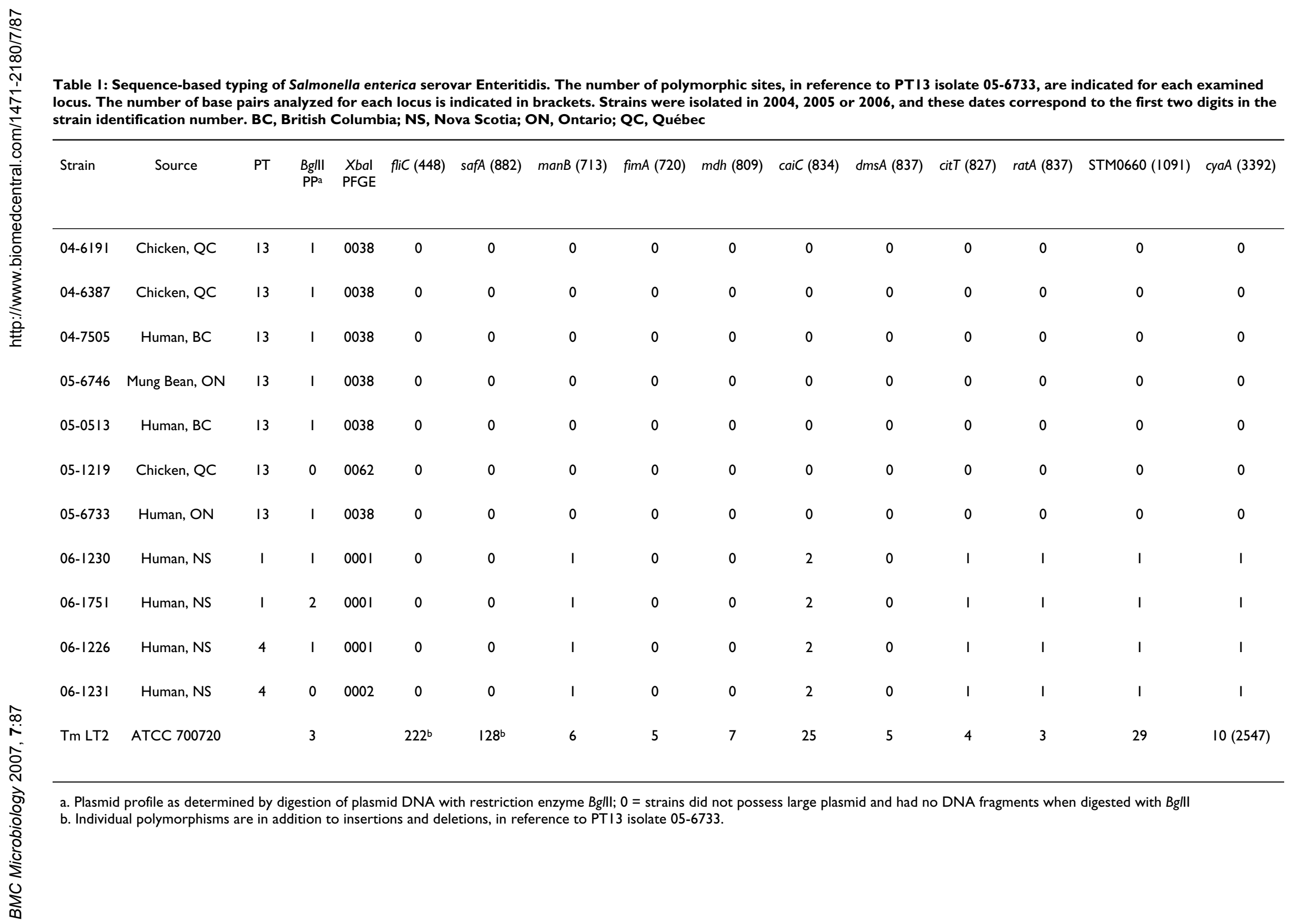




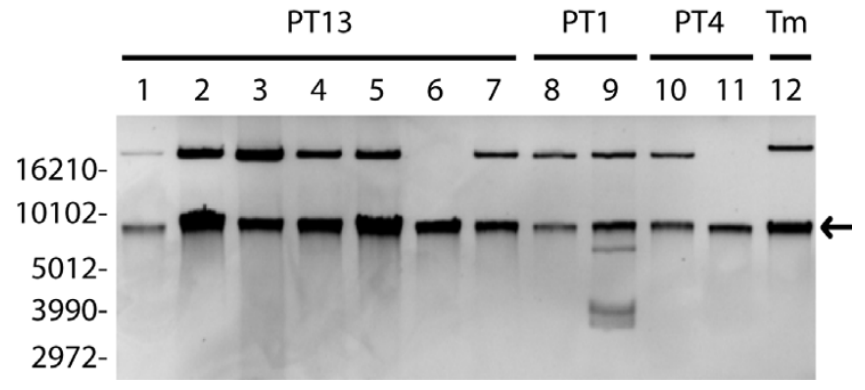

Figure 3

Plasmid profiles for $S$. Enteritidis strains used in this study. Preparations were not digested with restriction endonuclease. Lanes I-7: S. Enteritidis PTI 3 strains 04-6 I 9I, 04-6387, 04-7505, 05-6746, 05-05। 3, 05- I 219 and 05-6733 respectively. Lanes 8 and 9: S. Enteritidis PTI strains 06- 1230 and 06-I75I. Lanes 10 and II: S. Enteritidis PT4 strains 06-I2I6 and 06-I23I. Lane 12: plasmid extracted from S. Typhimurium LT2. Supercoiled DNA ladder molecular weights are to the left of lane I. Arrow indicates a chromosomal DNA fragment.

and in all plasmid preparations a chromosomal DNA fragment was observed (Fig. 3). A high molecular weight (HWM) plasmid was observed for all strains except PT13 strain 05-1219 and PT4 strain 06-1231. Accordingly, S. Typhimurium strain LT2 was known to harbor the $94 \mathrm{kbp}$ virulence plasmid pSLT [24] and S. Enteritidis commonly harbors a 60 kbp virulence plasmid [11]. Strains 051219 and 06-1231 that lacked the HMW virulence plasmid had a different PFGE pattern than the other $S$. Enteritidis PT13 and PT4 strains, respectively (Table 1 and Fig. $1)$. The only strain from which low molecular weight (LMW) plasmids were isolated was PT1 strain 06-1751. To confirm the presence of the Salmonella virulence plasmid in $S$. Enteritidis PT13, PCR screening for the $s p v$ C virulence determinant was performed. All strains encoded $s p v \mathrm{C}$ (data not shown) except for the two $S$. Enteritidis strains that did not posses the HMW virulence plasmid.

Restriction enzyme digestion of the plasmid preparations with $B g l I I$ revealed that $S$. Enteritidis strains harboring the HMW plasmid had the same restriction fragment length polymorphism (RFLP) pattern (Fig. 4). The S. Enteritidis PT1 strain with LMW plasmids had a different plasmid pattern due to additional DNA fragments (corresponding to the LMW plasmids) and the fragments in the pattern contributed by the HMW plasmid were otherwise identical to those of strains that contained only the HMW plasmid. Additionally, the RFLP pattern produced by the $\mathrm{S}$. Typhimurium pSLT differed from the $S$. Enteritidis HMW plasmid.

\section{Rep-PCR}

Repetitive sequence-based PCR (rep-PCR) methods targeting non-coding repetitive sequences are useful for bacterial subtyping and this platform has been automated and reproducibility improved through commerciallyavailable reagents in conjunction with a microfluidics station [33]. Automated rep-PCR was performed on S. Enteritidis PT13, PT1 and PT4 strains, and a small selection of other serovars was also included to illustrate the scope of differentiation at the serovar level. The rep-PCR amplicon patterns for all $S$. Enteritidis PT13 strains clustered together and were $>98 \%$ related with no significant differences (Fig. 5). The S. Enteritidis PT1 and PT4 strains produced similar rep-PCR patterns as the PT13 strains (>95\% relatedness) with a single amplicon being the predomi-

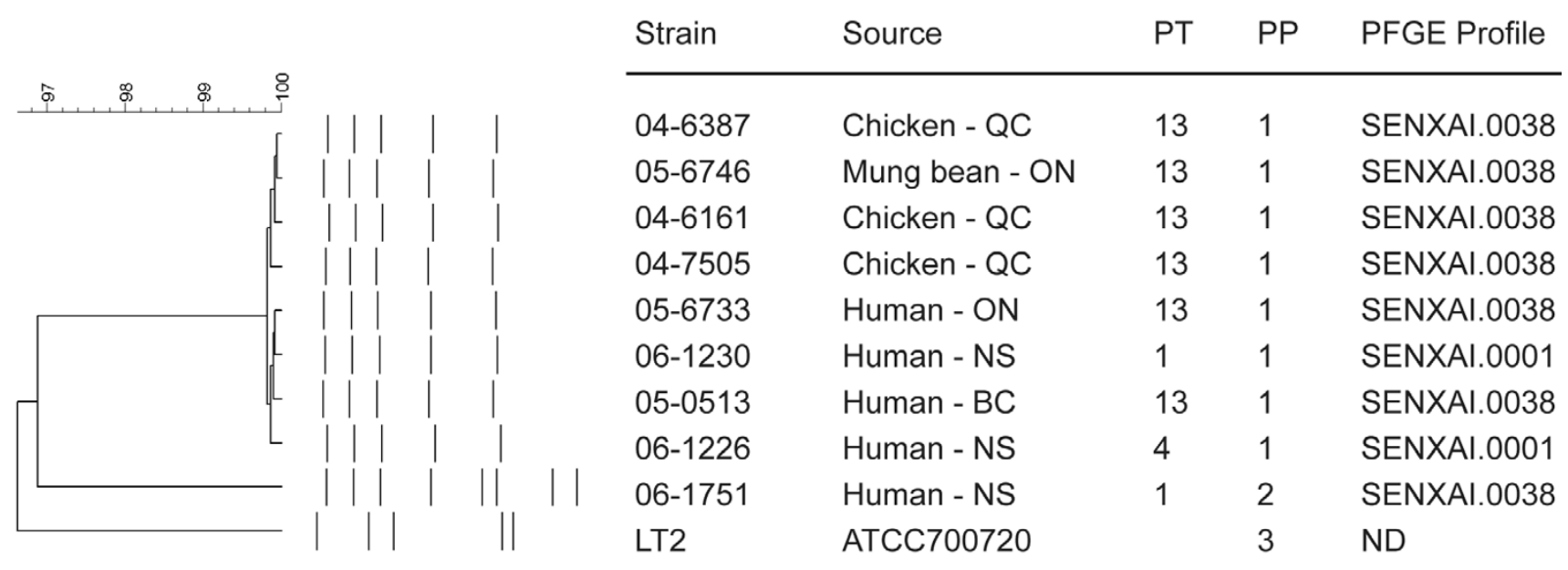

Figure 4

Plasmid RFLP patterns for S. Enteritidis PTI3, PTI and PT4 strains. Restriction fragment patterns generated with Bglll were analyzed in BioNumerics version 4 and a dendrogram was created using the UPGMA method with a coefficient of correlation, $2 \%$ optimization and $12 \%$ position tolerance. PP; RFLP plasmid pattern, PT; phage type. 
nant difference. The other examined serovars were between $78-81 \%$ related to the $S$. Enteritidis strains (Fig. $5)$.

\section{Discussion}

Genetic homogeneity has been repeatedly observed for $S$. Enteritidis, particularly between strains of the same phage type $[26,24,27]$. Similarly, subtyping beyond phage type utilizing methods such as ribotyping, PFGE and plasmid profiling has generally only confirmed the clonal lineages discerned by phage typing without providing any further discriminatory power $[10,34,29]$. This lack of subtyping can prevent the establishment of absolute links to contaminated agri-food sources during outbreak trace-back investigations. During an outbreak of $S$. Enteritidis PT13 in Canada, the outbreak strain could not be distinguished from concurrent and geographically and temporally distinct isolates strains of $S$. Enteritidis PT13. CGH, SBT, plasmid profiling and rep-PCR were performed in an attempt to identify genetic markers suitable for subtyping $S$. Enteritidis PT13.

Clonally related phenotypic subpopulations of $S$. Enteritidis have been observed and discrete allelic variation at ribosomal and metabolic loci corresponded to these

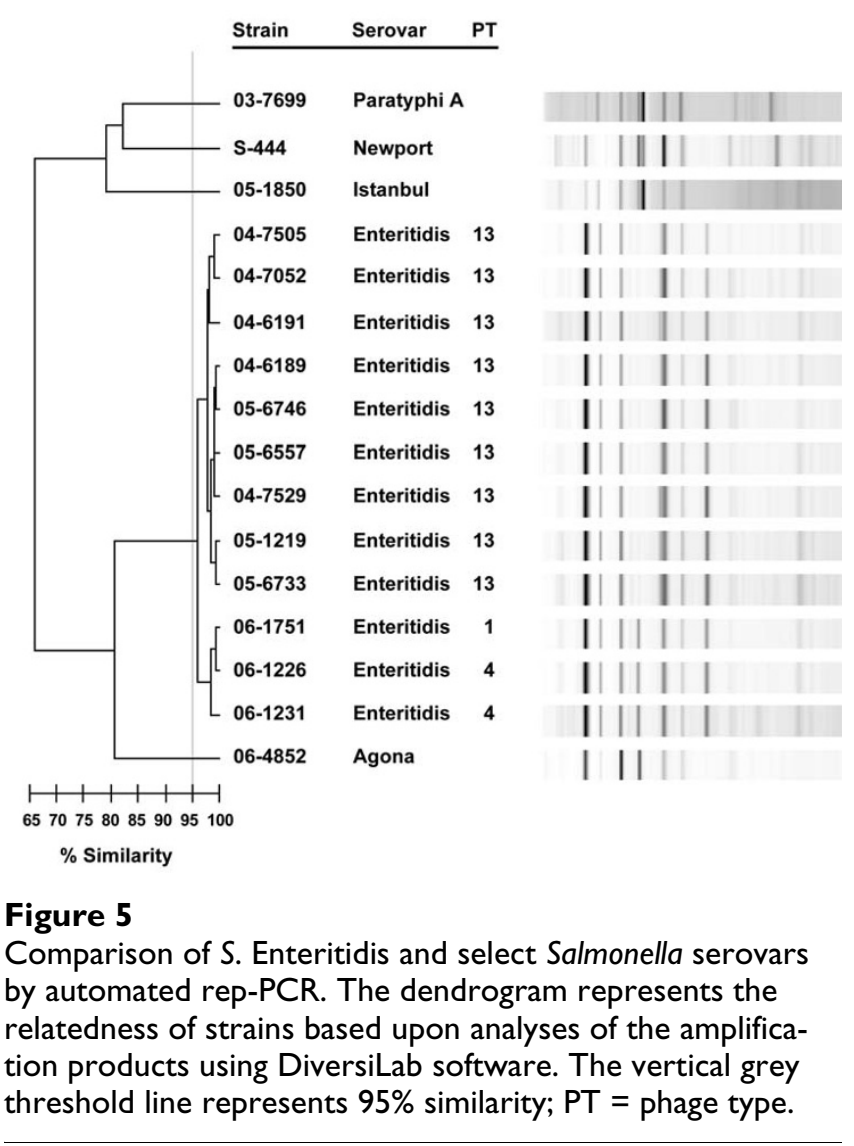

pathotypes [31,32]. In our CGH experiments, large clusters of genetic differences such as the absence of phage regions were discernible between $S$. Enteritidis and the reference $S$. Typhimurium for which the array was designed. However, more subtle genetic differences such as allelic variation between PT13 strains could not be confirmed, which was a finding similar to previous results [24,27]. This may have resulted from a lack of $S$. Enteritidis specific probes. Alternatively, identifying a reliable phenotypic or pathotype difference between PT13 strains could aid in the identification of subtle but definitive genetic differences that impact biology within a phage type.

Morales and colleagues hypothesized that genetic variation between $S$. Enteritidis strains is better studied by performing DNA sequencing for SNPs than through microarray-based comparative genomics [27]. Through sequencing, it is possible to produce de novo data for each strain instead of attempting to discern genomic content in the context of comparing hybridization data between two different strains, or possibly different serovars, as in this study ( $S$. Typhimurium reference DNA versus $S$. Enteritidis test DNA). Although DNA sequencing provided a higher level of detail than CGH, sequencing of the loci that were previously observed to be divergent between $S$. Enteritidis pathotypes and other loci commonly used for sequence-based typing (total 11,390 bp per strain) did not identify any genomic differences between PT13 strains. These results are similar to other published results in that discrimination between serovars was possible, but variation within a phage type was not observed $[29,26,35]$. Alternatively, some loci were significantly variable between $S$. Enteritidis and $S$. Typhimurium (LT2), including safA (128 SNP), STM0660 (29 SNP) and caiC (25 SNP). These variable loci may prove to be useful as targets for molecular typing between Salmonella serovars. Our panel of strains was selected to include the outbreakassociated strains, concurrent sporadic clinical and agrifood strains, and for comparison to unrelated strains, isolates that were previously recovered in different Canadian provinces. If the incidence of SNPs is less than 1 in 10,000 bp it may be necessary to pursue a whole genome approach for detection of allelic variation because any one section of the genome or group of genes could be similar between clonally related PT13 strains relevant to human public health.

Plasmid profile analyses identified $S$. Enteritidis strains that lacked the HMW virulence plasmid or those that contained LMW plasmids, but these did not correspond to the outbreak-associated strains. Carriage of the HMW virulence plasmid were confirmed by testing for the presence of the $s p v C$ gene, which was previously observed on all $S$. Enteritidis plasmids of $\sim 60 \mathrm{kbp}[16]$. The variation in plasmid content did influence the observed XbaI PFGE 
patterns, which were otherwise identical. Additionally, RFLP analyses of the plasmid preparations did not identify any further levels of genetic diversity within the plasmids, but did support that both $S$. Enteritidis PT1 and PT4 carry a similar HMW virulence plasmid as $S$. Enteritidis PT13. Furthermore, the usefulness of determining plasmid carriage as a means of subtyping was limited since the overall incidence of the HMW virulence plasmids in Canada was unknown and variation of this trait may be influenced by laboratory conditions (i.e. plasmid loss during culturing).

Rep-PCR has the potential to represent genetic differences contributed throughout the genome, unlike directed SBT and plasmid profiling, and targets different genetic features than PFGE. The S. Enteritidis PT13 strains examined in this study were not differentiated with this method and were greater than $97 \%$ related. Although there was some discrimination between the S. Enteritidis PT1 and PT4 strains and the $S$. Enteritidis PT13 strains (by a single band difference), there was still greater than $95 \%$ relatedness amongst these strains. Alternatively, a select number of other serovars were examined using automated rep-PCR and this method could differentiate between serovars, which is similar to a previous rep-PCR study [36].

\section{Conclusion}

$S$. Enteritidis PT13 isolates in Canada were observed to be essentially genetically homogeneous after microarraybased comparisons did not identify overt differences between strains and DNA sequencing of eleven loci and 11,390 bp did not identify any polymorphisms. Furthermore, PFGE and plasmid RFLP patterns were identical for the majority of strains, except for some changes in plasmid content that affected restriction patterns of strains not associated with a large foodborne outbreak. Rep-PCR could discriminate between serovars and phage types, but not between outbreak-related and sporadic PT13 isolates. The apparent clonality of PT13 strains has implications for the ability to subtype this pathogen during outbreaks.

\section{Methods \\ Bacterial strains}

Salmonella enterica serovar Enteritidis strains were isolated from human, food and animal sources by public health laboratories in Ontario, Québec, British Columbia and Nova Scotia during 2004-2006 and submitted to the Enteric Diseases Program at the National Microbiology Laboratory. S. enterica serovar Typhimurium LT2 (ATCC 700720) was included as a positive control for CGH and SBT because many of the utilized oligonucleotides were designed using the genomic sequence data from this strain (GenBank accession number NC 003197). Confirmation of phage type was completed with bacteriophage stocks prepared at the National Microbiology Laboratory.
Antibiotic sensitivity testing was completed using the National Antimicrobial Resistance Monitoring System (NARMS) recommended panel and the Sensititre broth dilution method.

\section{Plasmid Isolation and Analysis}

Plasmids were purified from overnight culture using a Qiagen plasmid Midi kit (Qiagen, Mississauga, ON) according to manufacturer's directions with the following modification: plasmids were precipitated using $7.5 \mathrm{M}$ ammonium acetate (Sigma-Aldrich, St. Louis, MO) in combination with isopropanol. Purified plasmid DNA $(25 \mu \mathrm{l})$ was digested overnight at $37^{\circ} \mathrm{C}$ with 20 units of BglII (New England Biolabs, Pickering, ON). Resulting plasmid fragments were separated by electrophoresis on $0.7 \%$ Tris-acetate-EDTA agarose gels at $70 \mathrm{~V}$ for 6 hours in $1 \times$ Tris-acetate-EDTA buffer (Gibco BRL, Paisley, Scotland). A $1 \mathrm{~Kb}$ Plus DNA Ladder (Invitrogen) and TrackIt ${ }^{\mathrm{TM}}$ $\lambda$ DNA/HindIII fragments (Invitrogen) were used as molecular size standards. Gels were stained with ethidium bromide $(2 \mu \mathrm{g} / \mathrm{ml})$ and digital images were obtained using a Bio-Rad Gel Doc XR (Bio-Rad).

\section{PCR and Sequencing}

Template DNA was prepared by centrifuging $1 \mathrm{~mL}$ of logphase cultures grown in brain heart infusion (BHI) broth, the pellet was resuspended in $1 \mathrm{~mL}$ of TE buffer (Sigma, 10 mM Tris-HCl, 1 mM EDTA, pH 8.0) and boiling for 15 minutes. Boiled cells were pelleted and the supernatant was removed and used as DNA template in PCR reactions.

Oligonucleotide primer sequences used for DNA amplification and/or sequencing are presented in Table 2. PCR was performed with Platinum Taq (Invitrogen), following the manufacturer's directions. The thermocycling parameters for fim $A, m a n B$ and $m d h$ included an initial denaturation at $94^{\circ} \mathrm{C}$ for 5 minutes, 35 cycles of denaturation at $94^{\circ} \mathrm{C}$ for 30 seconds, annealing at $50^{\circ} \mathrm{C}$ for 30 seconds and extension at $68^{\circ} \mathrm{C}$ for 45 seconds, with a final extension at $68^{\circ} \mathrm{C}$ for 5 minutes. The annealing temperature for caiC, dms A, cit T, ratA, STM0660, was $55^{\circ} \mathrm{C}$ for 40 seconds and an extension of 60 seconds. The annealing temperature for safA and $f l i C$ was $50^{\circ} \mathrm{C}$ for 30 seconds and an extension of 30 seconds. For amplification of cyaA, the annealing temperature was $55^{\circ} \mathrm{C}$ for 60 seconds with an extension of 120 seconds. PCR products were purified using the QIAquick PCR purification kit (Qiagen) and sequenced using the same primers that generated these amplicons, with the exception of $c y a A$ that required ten additional oligonucleotides targeting internal segments to provide complete coverage (Table 2). Sequencing was performed with an ABI3730 capillary electrophoresis instrument (Applied Biosystems, Foster City, CA). Multiple sequence alignments were completed using ClustalW [37] 
Table 2: Oligonucleotides used in this study. For oligonucleotides used in PCR the amplicon size is listed, and for those oligonucleotides used solely for DNA sequencing no product size is listed

\begin{tabular}{|c|c|c|c|c|}
\hline Oligonucleotide & Target & Sequence (5' to $\left.3^{\prime}\right)$ & Product size (bp) & Reference \\
\hline sfimAF & fimA & TCAGGGGAGAAACAGAAAACTAAT & 760 & {$[25]$} \\
\hline sfimAR & & TCCCCGATAGCCTCTTCC & & {$[25]$} \\
\hline smanBF & $\operatorname{man} B$ & CATAACCCGATGGACTACAACG & 893 & {$[25]$} \\
\hline smanBR & & ACCAGCAGCCACGGGATCAT & & {$[25]$} \\
\hline smdhF & $m d h$ & GATGAAAGTCGCAGTCCTCG & 849 & {$[25]$} \\
\hline smdhR & & TATCCAGCATAGCGTCCAGC & & {$[25]$} \\
\hline $\mathrm{ccmBF}$ & $\mathrm{ccm} B$ & TCACCCTGTTTCCGTTAAGC & 430 & This study \\
\hline $\mathrm{ccmBR}$ & & AAAATCAGCACCGGGACAC & & This study \\
\hline umuCF & umuC & TGACCACACTCGAGGAGATG & 493 & This study \\
\hline umuCR & & CAAACGATTTCCTGCTTTGC & & This study \\
\hline fljAF & $f j A$ & GGCGAGAAGCTGAAATATGG & 410 & This study \\
\hline fljAR & & ATTTACGCCTGTCGTTTTGC & & This study \\
\hline safA2F & safA & TAAGAGGTGCTCTGATATATAG & 959 & This study \\
\hline safA2R & & ATAGGGTAATTCTGCGGGTTG & & This study \\
\hline caiCIF & caic & GAATCGTTCGGCAGTTTAGC & 874 & This study \\
\hline caiCIR & & GTTTCAGTCATACCATAAGAGG & & This study \\
\hline citTIF & citT & GATGATTGTCGGTATGATCC & 870 & This study \\
\hline citTIR & & GTAATATCTTTCCACGGCAC & & This study \\
\hline dmsAIF & $d m s A$ & ACTACGGTGATTACTCTTCC & 869 & This study \\
\hline dmsAIR & & CTGGTTAATCAAACAGTTGC & & This study \\
\hline $\operatorname{ratAIF}$ & ratA & GGCAAGATTCACAGCATTCAG & 870 & This study \\
\hline $\operatorname{rat} A I R$ & & TGGGCGGTATTTATCGTTCG & & This study \\
\hline STM0660F & STM0660 & ACGATGTAGCCCATATTACG & 1191 & This study \\
\hline STM0660R & & CCTGGCGAAAGTATTCATCC & & This study \\
\hline $\mathrm{spvCl}$ & $\operatorname{spvC}$ & AАCTCCTTGCACAACCAAATG & 230 & This Study \\
\hline spvC2 & & ACCATATCCCTGAGCACACTG & & This Study \\
\hline CyalsF & cyaA & CATTGACCATCCTAACATCCTTATAGAGAG & 3331 & [3I] \\
\hline Cya6sR & & ACTGGCGATATCACTCAATAGCGG & & {$[31]$} \\
\hline CyalsR & cyaA & ATGCTGCGTAGAACCACAGTCTTC & & {$[31]$} \\
\hline Cya2sF & cyaA & TGGATATCTGGGTGTGCCATCAGT & & [3I] \\
\hline Cya2sR & cyaA & GACTTTACCCGGCAACGCTTCAAA & & [31] \\
\hline Cya3sF & cyaA & CGATTACCGGCGTTTACACCAT & & {$[31]$} \\
\hline Cya3sR & cyaA & ATATCTTTCGCCAGCAGACGTG & & {$[31]$} \\
\hline Cya4sF & cyaA & CGCAGGATATCGGCGTACTGA & & {$[31]$} \\
\hline Cya4sR & cyaA & GACGGCAATTTCACCTGGTGGTT & & {$[31]$} \\
\hline Cya5sF & cyaA & GCGTCGGGAAGTATTAAGCCAGTT & & {$[31]$} \\
\hline Cya5sR & cyaA & AGGTCGACAATACCGTTGCCCTTA & & [3I] \\
\hline Cya6sF & cyaA & TACGTCTTCCAGCACCCGTCA & & [31] \\
\hline
\end{tabular}

and Boxshade [38], and these data were deposited in GenBank with accession numbers EF113924-EF113956.

\section{Rep-PCR typing}

Total bacterial genomic DNA was isolated from selected SE strains (Table 2) and a small selection of other Salmonella serovars ( $S$. Agona strain 06-4852, S. Istanbul 051850, S. Newport S-444, and S. Paratyphi A 03-7699) using the Qiagen DNeasy extraction kit, following manufacturer's instructions. The DiversiLab Salmonella DNA fingerprinting kit (Bacterial Barcodes) was used for automated rep-PCR molecular typing using AmpliTaq DNA polymerase (Roche) and PCR conditions identified by Bacterial Barcodes. The rep-PCR amplicons were separated using an Agilent 2100 Bioanalyzer on the DiversiLab microfluidics DNA chip. DNA fingerprints were statistically analyzed using DiversiLab software using manufacturer's instructions.

\section{Comparative Genomic Hybridizations}

DNA microarrays were constructed as previously described [39] from 4451 commercially-supplied oligonucleotides (Qiagen) representing coding sequences of $S$. Typhimurium LT2, with the custom addition of the putative open reading frames from SGI1. Each oligonucleotide was spotted in duplicate per slide. DNA was isolated from overnight cultures grown in $\mathrm{BHI}$ broth at $37^{\circ} \mathrm{C}$ using the alkaline lysis protocol [40]. Proteins were removed using phenol chloroform extractions in Phase lock gel tubes (Eppendorf, Westbury, NY) and purified DNA was frag- 
mented in a Nebulizer (Invitrogen) as per manufacturer's protocols, with the exception that DNA was nebulized for 3 minutes at 10 psi to yield DNA fragments between 500 and $700 \mathrm{bp}$. Probe DNA was created by labeling the fragmented genomic DNA with Cy3- or Cy5-dCTP (Amersham, Baie d'Urfe, QC) by random priming using the Bioprime Labeling Kit (Invitrogen) following the manufacturer's directions. The concentration and labeling efficiencies were measured using the NanoDrop ND-1000 spectrophotometer (NanoDrop technologies, Wilmington, DE). For each array a Cy3 or Cy5-labeled reference probe DNA (always $S$. Typhimurium LT2) was combined with a Cy3 or Cy5-labeled test PT13 strain along with 20 $\mu \mathrm{g}$ of Salmon Sperm DNA and dried in a Vacufuge (Eppendorf). Each test-versus-reference comparison was performed in triplicate, with at least one of the slides hybridized as a dye swap. The DNA pellet was resuspended in $10 \mu \mathrm{l}$ of $\mathrm{ddH}_{2} \mathrm{O}$, denatured at $95^{\circ} \mathrm{C}$ for $5 \mathrm{~min}$ utes, and snap cooled on ice for 5 minutes prior to hybridization to the array.

Slides were prepared as previously published [39] with the exception that they were pre-hybridized at $42^{\circ} \mathrm{C}$ in DIG Easy-Hyb buffer (Roche Diagnostic, Laval, QC) for 45 minutes, followed by brief washes in $\mathrm{dH}_{2} \mathrm{O}$ and isopropanol. $50 \mu \mathrm{L}$ of DIG Easy-Hyb buffer was added to each probe mixture and the entire volume was pipetted under an M-Series LifterSlip ${ }^{\mathrm{TM}}$ (Erie Scientific Co., Portsmouth, $\mathrm{NH}$ ) onto the pre-hybridized slides. Slides were hybridized at $42^{\circ} \mathrm{C}$ in a hybridization chamber (Genetix, Hampshire, UK). Slides were sequentially washed as follows: Buffer 1 ( $1 \times$ SSC and $0.2 \%$ SDS: Amersham) for 6 minutes at $56^{\circ} \mathrm{C}$; Buffer $2(0.1 \times$ SSC and $0.2 \%$ SDS $)$ for 4 minutes at room temperature; two washes for 2 minute with Buffer $3(0.1 \times$ SSC). Slides were spun dry and scanned using the Agilent DNA microarray scanner (Agilent Technologies, Mississauga, $\mathrm{ON}$ ).

Scanned slide images were analyzed as previously published [39] with the exception that following normalization and batch-effect removal, the data was antilog $2_{2}^{-}$ transformed to convert back to its original scale so that the $\log _{2}$ ratio between test and reference could be measured. Log ratios were averaged across all replicates per spot for each test versus reference comparison. GeneMaths XT software (Applied Maths, Austin, TX) was used for Hierarchical clustering of the data to identify overall genetic relatedness between examined strains, and identify specific loci that were putatively absent of divergent in individual $S$. Enteritidis strains.

\section{Abbreviations}

Comparative genomic hybridization (CGH), High molecular weight (HMW), Low molecular weight (LMW), Phage type (PT), Pulsed field gel electrophoresis (PFGE), Restric- tion fragment length polymorphism (RFLP), RFLP Plasmid pattern (PP), Salmonella enterica serovar (S.), Sequence based typing (SBT), Single nucleotide polymorphism (SNP).

\section{Competing interests}

The author(s) declares that there are no competing interests.

\section{Authors' contributions}

ABO performed CGH, SBT and plasmid profile analyses for this study, assisted in the study design, and drafted the manuscript. AKA and DMT participated in plasmid isolations and analysis, rep-PCR analysis, and assisted in drafting and revising the manuscript. JGB provided access to whole genomic analysis of $S$. Enteritidis subpopulations and assisted in the sequence analysis. WD, AM, FJ and LKN assisted in conceiving the study, provided epidemiological data for strains and revised the manuscript. MWG supervised the project and drafted and revised the manuscript. All authors read and approved the final manuscript.

\section{Acknowledgements}

We would like to thank Michael Mulvey for providing oligonucleotides for the Salmonella microarray, Martin Wiedmann for sharing Salmonella SBT data, PulseNet Canada (Cynthia Misfeldt, Lorelee Tschetter and Jason Allen) for PFGE analysis, Melissa McCracken for technical help and Helen Tabor and Clifford Clark for helpful discussions. We also thank the Canadian Integrated Program from Antimicrobial Resistance Surveillance (CIPARS), British Columbia Centre for Disease Control, Nova Scotia Public Health Laboratory and the Laboratoire de Sante Publique du Québec for providing strains. Oligonucleotide synthesis, DNA sequencing and array printing was performed by the DNA Core facility and phage typing was performed by the Enteric Diseases Program at the National Microbiology Laboratory.

\section{References}

I. St Louis ME, Morse DL, Potter ME, DeMelfi TM, Guzewich J], Tauxe $\mathrm{RV}$, Blake PA: The emergence of grade $A$ eggs as a major source of Salmonella enteritidis infections. New implications for the control of salmonellosis. JAMA 1988, 259:2103-2107.

2. Poppe C: Epidemiology of Salmonella enterica Serovar Enteritidis. In Salmonella enterica Serovar Enteritidis in Humans and Animals Edited by: Saeed AM, Gast RK, Potter ME and Wall PG. Ames, lowa State University Press; 1999:3-I8.

3. Munro DS, Girdwood RWA, Reilly W]: Salmonella enterica Serovar Enteritidis in Scotland. In Salmonella enterica Serovar Enteritidis in Humans and Animals Edited by: Saeed AM, Gast RK, Potter ME and Wall PG. Ames, lowa State University Press; 1999:27-3I.

4. Cogan TA, Humphrey TJ: The rise and fall of Salmonella Enteritidis in the UK. J Appl Microbiol 2003, 94 Suppl: I I4S- I I 9S.

5. Tschape H, Liesegang A, Gericke B, Prager R, Rabsch W, Helmuth R: Ups and Downs of Salmonella enterica Serovar Enteritidis in Germany. In Salmonella enterica Serovar Enteritidis in Humans and Animals Edited by: Saeed AM, Gast RK, Potter ME and Wall PG. Ames, lowa State University Press; 1999:5I-6I.

6. Mishu B, Koehler J, Lee LA, Rodrigue D, Brenner FH, Blake P, Tauxe RV: Outbreaks of Salmonella enteritidis infections in the United States, I 985-I 99 I. J Infect Dis 1994, I 69:547-552.

7. Angulo FJ, Swerdlow DL: Epidemiology of Human Salmonella enterica Serovar Enteritidis Infections in the United States. In Salmonella enterica Serovar Enteritidis in Humans and Animals Edited 
by: Saeed AM, Gast RK, Potter ME and Wall PG. Ames, lowa State University Press; 1999:33-4I.

8. Demczuk W, Woodward D, Ahmed R, Clark C, Tabor H, Dore K Ciampa N, Muckle A: Laboratory surveillance data for enteric pathogens in Canada: Annual summary 2002 and 2003. Winnipeg, MB, Health Canada; 2005.

9. Ward LR, de Sa JD, Rowe B: A phage-typing scheme for Salmonella Enteritidis. Epidemiol Infect 1987, 99:29I-294.

10. Thong KL, Ngeow YF, Altwegg M, Navaratnam P, Pang T: Molecular analysis of Salmonella Enteritidis by pulsed-field gel electrophoresis and ribotyping. J Clin Microbiol 1995, 33:1070-1074.

II. Pang JC, Chiu TH, Chiou CS, Schroeter A, Guerra B, Helmuth R, Tsen HY: Pulsed-field gel electrophoresis, plasmid profiles and phage types for the human isolates of Salmonella enterica serovar Enteritidis obtained over I3 years in Taiwan. J Appl Microbiol 2005, 99: | 472-I483.

12. Millemann Y, Lesage MC, Chaslus-Dancla E, Lafont JP: Value of plasmid profiling, ribotyping, and detection of IS200 for tracing avian isolates of Salmonella Typhimurium and S. Enteritidis. J Clin Microbiol 1995, 33: I73-I 79.

13. Liebana E, Garcia-Migura L, Breslin MF, Davies RH, Woodward MJ: Diversity of strains of Salmonella enterica serotype Enteritidis from English poultry farms assessed by multiple genetic fingerprinting. J Clin Microbiol 200I, 39:154-161.

14. Liebana E, Garcia-Migura L, Clouting C, Clifton-Hadley FA, Breslin M, Davies RH: Molecular fingerprinting evidence of the contribution of wildlife vectors in the maintenance of Salmonella Enteritidis infection in layer farms. J Appl Microbiol 2003. 94: 1024-1029.

15. Liebana E, Clouting C, Garcia-Migura L, Clifton-Hadley FA, Lindsay E, Threlfall EJ, Davies RH: Multiple genetic typing of Salmonella Enteritidis phage-types 4, 6, 7, 8 and 13 a isolates from animals and humans in the UK. Vet Microbiol 2004, 100:189-195.

16. Soto SM, Rodriguez I, Rodicio MR, Vila J, Mendoza MC: Detection of virulence determinants in clinical strains of Salmonella enterica serovar Enteritidis and mapping on macrorestriction profiles. I Med Microbiol 2006, 55:365-373.

17. Clark CG, Kruk TM, Bryden L, Hirvi Y, Ahmed R, Rodgers FG: Subtyping of Salmonella enterica serotype Enteritidis strains by manual and automated Pstl-Sphl ribotyping. J Clin Microbiol 2003, 4I:27-33.

18. Bakeri SA, Yasin RM, Koh YT, Puthucheary SD, Thong KL: Genetic diversity of human isolates of Salmonella enterica serovar Enteritidis in Malaysia. J Appl Microbiol 2003, 95:773-780.

19. Ahmed R, Soule G, Demczuk WH, Clark C, Khakhria R, Ratnam S, Marshall S, Ng LK, Woodward DL, Johnson WM, Rodgers FG: Epidemiologic typing of Salmonella enterica serotype Enteritidis in a Canada-wide outbreak of gastroenteritis due to contaminated cheese. J Clin Microbiol 2000, 38:2403-2406.

20. Ridley AM, Threlfall EJ, Rowe B: Genotypic characterization of Salmonella enteritidis phage types by plasmid analysis, ribotyping, and pulsed-field gel electrophoresis. J Clin Microbiol 1998, 36:23|4-232|.

21. Powell NG, Threlfall EJ, Chart H, Rowe B: Subdivision of Salmonella enteritidis $P T \mathbf{4}$ by pulsed-field gel electrophoresis: potential for epidemiological surveillance. FEMS Microbiol Lett 1994, II 19:193-198.

22. Landeras E, Gonzalez-Hevia MA, Alzugaray R, Mendoza MC: Epidemiological differentiation of pathogenic strains of Salmonella Enteritidis by ribotyping. J Clin Microbiol 1996, 34:2294-2296.

23. Liebisch B, Schwarz S: Molecular typing of Salmonella enterica subsp. enterica serovar Enteritidis isolates. J Med Microbiol 1996, 44:52-59.

24. Porwollik S, Santiviago CA, Cheng P, Florea L, McClelland M: Differences in gene content between Salmonella enterica serovar Enteritidis isolates and comparison to closely related serovars Gallinarum and Dublin. J Bacteriol 2005, I87:6545-6555.

25. Tassios PT, Markogiannakis A, Vatopoulos AC, Katsanikou E, Velonakis EN, Kourea-Kremastinou J, Legakis NJ: Molecular epidemiology of antibiotic resistance of Salmonella Enteritidis during a 7-year period in Greece. J Clin Microbiol 1997, 35:1316-132|.

26. Sukhnanand S, Alcaine S, Warnick LD, Su WL, Hof J, Craver MP, McDonough P, Boor KJ, Wiedmann M: DNA sequence-based sub- typing and evolutionary analysis of selected Salmonella enterica serotypes. J Clin Microbiol 2005, 43:3688-3698.

27. Morales CA, Porwollik S, Frye JG, Kinde H, McClelland M, GuardBouldin J: Correlation of phenotype with the genotype of eggcontaminating Salmonella enterica serovar Enteritidis. Appl Environ Microbiol 2005, 7 I:4388-4399.

28. Kotetishvili M, Stine OC, Kreger A, Morris JG Jr., Sulakvelidze A Multilocus sequence typing for characterization of clinical and environmental Salmonella strains. J Clin Microbiol 2002, 40:1626-1635.

29. Hudson CR, Garcia M, Gast RK, Maurer JJ: Determination of close genetic relatedness of the major Salmonella enteritidis phage types by pulsed-field gel electrophoresis and DNA sequence analysis of several Salmonella virulence genes. Avian Dis 200I, 45:875-886.

30. Boyd D, Peters GA, Cloeckaert A, Boumedine KS, Chaslus-Dancla E, Imberechts H, Mulvey MR: Complete nucleotide sequence of a 43-kilobase genomic island associated with the multidrug resistance region of Salmonella enterica serovar Typhimurium DTI 04 and its identification in phage type DTI20 and serovar Agona. J Bacteriol 200I, I 83:5725-5732.

31. Morales CA, Musgrove M, Humphrey TJ, Cates C, Gast R, GuardBouldin J: Pathotyping of Salmonella enterica by analysis of single-nucleotide polymorphisms in cyaA and flanking $23 \mathrm{~S}$ ribosomal sequences. Environ Microbiol 2007, 9:1047-1059.

32. Comparative analyses of $\mathbf{S}$. Enteritidis phenotypic subpopulations 2007 [http://www.ncbi.nlm.nih.gov/genomes/staticl Salmonella SNPS.html].

33. Healy M, Huong J, Bittner T, Lising M, Frye S, Raza S, Schrock R, Manry J, Renwick A, Nieto R, Woods C, Versalovic J, Lupski JR: Microbial DNA typing by automated repetitive-sequencebased PCR. I Clin Microbiol 2005, 43:199-207.

34. Olsen JE, Skov MN, Threlfall EJ, Brown DJ: Clonal lines of Salmonella enterica serotype Enteritidis documented by IS200-, ribo-, pulsed-field gel electrophoresis and RFLP typing. J Med Microbiol 1994, 40: 15-22.

35. Fakhr MK, Nolan LK, Logue CM: Multilocus sequence typing lacks the discriminatory ability of pulsed-field gel electrophoresis for typing Salmonella enterica serovar Typhimurium. J Clin Microbiol 2005, 43:221 5-2219.

36. Bennasar A, de Luna G, Cabrer B, Lalucat J: Rapid identification of Salmonella typhimurium, $\mathbf{S}$. enteritidis and $\mathbf{S}$. virchow isolates by polymerase chain reaction based fingerprinting methods. Int Microbiol 2000, 3:31-38.

37. ClustalW alignment tool 2007 [http://www.ebi.ac.uk/clustalw/].

38. Boxshade 2007 [http://www.ch.embnet.org]

39. Golding GR, Olson AB, Doublet B, Cloeckaert A, Christianson S, Graham MR, Mulvey MR: The effect of the Salmonella genomic island $I$ on in vitro global gene expression in Salmonella enterica serovar Typhimurium LT2. Microbes Infect 2007, 9:21-27.

40. Sambrook J, Russell DW: Molecular Cloning: A Laboratory Manual Volume I. 3rd edition. Cold Spring Harbour, Cold Spring Harbour Laboratory Press; 200I:I.32-I.34.
Publish with Biomed Central and every scientist can read your work free of charge

"BioMed Central will be the most significant development for disseminating the results of biomedical research in our lifetime. "

Sir Paul Nurse, Cancer Research UK

Your research papers will be:

- available free of charge to the entire biomedical community

- peer reviewed and published immediately upon acceptance

- cited in PubMed and archived on PubMed Central

- yours - you keep the copyright
BioMedcentral 